\title{
Producing Lecture Videos from Face-to-Face Teaching
}

\author{
Mikko Myllymäki, Jari Penttilä, and Ismo Hakala
}

\begin{abstract}
There is a clear demand for lecture videos in modern education. Nevertheless, the desire to keep face-to-face education remains as well. In such a case, the natural option is to produce videos in face-to-face teaching situations rather than in a studio environment. The challenge this kind of production faces is how to make it to cover an entire degree program cost-efficiently while serving the purpose intended. This article explains the demands set for video production in the context of face-to-face education at the scale of a degree program and how to effectively respond to them in practice. The paper also evaluates the effectiveness and impacts of the production model developed in connection with the Master Studies in Mathematical Information Technology at the Kokkola University Consortium Chydenius. According to the results, with the help of a recording system satisfying the requirements of the education provider it is possible to produce lecture videos at the scale of a degree program, cost-efficiently and in a way that reliably provides support for study.
\end{abstract}

Index Terms-Blended learning, lecture videos, video production.

\section{INTRODUCTION}

Technological development together with the speeding up of data communications and their widespread presence have, among other things, enabled video that can be distributed through networks. Today, videos are produced for a variety of purposes. One common purpose of video production which includes many special features is that for educational use. A lot is expected from modern education in the form of e.g. flexibility and study methods customized for individuals. In many educational institutes, also in universities, clear needs have arisen for increasing flexibility in education with the utilization of video technologies.

Videos meant for educational use, in particular, have many special features that are challenging from the viewpoint of video production. Meeting these challenges has often been seen so exacting that introduction of videos at a larger scale is regarded sometimes as impossible even in spite of the benefits they can provide.

Videos for educational use can be produced either in studios or live in face-to-face teaching situations. Studio production is expensive and requires a great deal of work, especially if it is intended to be offered also as face-to-face teaching. Preserving face-to-face teaching alongside with videos brings some benefits when compared to teaching realized with videos only. It for example gives the students a

Manuscript received May 23, 2013; revised September 9, 2013. This work was supported by the European Commission and The European Regional Development Fund.

The authors are with the Kokkola University Consortium Chydenius, University of Jyväskylä, Finland (e-mail: mikko.myllymaki@chydenius.fi, jari.penttila@chydenius.fi, ismo.hakala@chydenius.fi). choice in the participation mode among face-to-face learning, real-time video transmissions and on-demand videos and thus supports a variety of learning styles. It also provides an opportunity to attach interactivity to video lectures. For these reasons, it is often seen desirable to produce videos directly from face-to-face teaching situations.

Producing lecture videos from face-to-face teaching is challenging if the intention is that the production should cover the teaching for an entire degree program and not just individual lectures. This would require that videos would have to be simultaneously produced e.g. from several session-related locations to ensure the production's suitability for different courses and lecturers. Extensive productions also face the challenge of keeping the practices associated with quality production cost-efficient. In addition to challenges faced by video production in connection with face-to-face teaching, there are more demands for transparency and visibility of the material presented: video production should not distract face-to-face teaching sessions, and it should provide distance students with a starting point for study as similar as possible to face-to-face teaching.

There are numerous different commercial products that make the production process of videos easier. There are also recording solutions especially designed for teaching use: e.g., PanOpto, SonicFoundry, vBrick and echo360. These commercial recording solutions typically provide software for recording and real-time transmission. In some of the systems, video distribution can be integrated into a learning management system (LMS). Often, commercial solutions provide administrative features that make the timing of recordings easier. Some systems may also provide support for mobile equipment. However, in the case of PanOpto for example, this applies only to iPhone and iPad equipment. Commercial recording solutions have, in addition, manufacturer-specific features, which may be useful in some cases. These include the commenting option as well as various activation choices for the viewers of video.

For some types of use, the commercial recording solutions may be suitable almost out of box. Typically, it is nevertheless difficult to find a turnkey production system that would, comprehensively and without compromises, meet the many challenges presented above. Often the most functional solution is to build one's own production system by utilizing, combining and complementing various equipment and software solutions available. A system created in this way suits the best way possible to the special features of the education concerned and integrates optimally to the already existing systems. It is also easier to construct functional practices for video production around a recording system developed from the producer's own perspective.

Typically, commercial recording solutions include only the required software; in addition, the equipment must still be 
acquired. There are large variations in the prices of commercial systems, reflecting the intended extent of recording. The greater the number of concurrent recording points and users, the higher the price is. If the system is made to suit the producer's own needs by utilizing and combining various commercial equipment and software solutions, it is possible to develop the system economically in smaller steps as the requirements grow. This kind of autonomously constructed recording system is considerably better for circumstances where big one-time investments are out of question.

Earlier research related to lecture recordings has primarily focused on the impacts of recording from the viewpoints, e.g., of learning outcomes [1]-[3], amounts of use [4], [5], reasons for use [3], [6] and participation [1], [7]. It is harder to find research on implementation of actual recording systems. Moreover, in these studies the production process and recording system itself has been evaluated very little or not at all [4], [6], [8], [9].

This paper deals with the challenges of lecture video production in connection with face-to-face teaching and presents a production model as well as technical solutions suitable for it. The functionality of the model presented is evaluated with the help of data collected in connection with the Master Studies in Mathematical Information Technology at the Kokkola University Consortium Chydenius.

\section{Video Production From A FACE-TO-FACE TEACHING SITUATION}

This chapter presents some video production challenges and some special features of production carried out in face-to-face teaching situations particularly. This is followed by a presentation of a production process for lecture videos from a face-to-face teaching situation and the technological solutions that accompany it.

\section{A. Production Challenges}

There are several challenges associated with the production of videos meant for study purposes. Many of these challenges become emphasized especially when it is seen desirable to produce videos directly from a face-to-face teaching situation.

Naturally, one would hope that video production wouldn't distract the face-to-face teaching session. Thus, the production must be as transparent as possible when a video is produced from a real face-to-face teaching situation. Transparency here means that the lecturer can concentrate on lecturing without having to significantly adapt the teaching and that the technology involved wouldn't disturb and divert attention from the teaching. It is also hoped that the technology and recording would not distract the students participating in face-to-face teaching.

The aim in making lecture videos available is to provide students who study with the help of video with starting points that are equal to those of face-to-face students. For this reason, the general approach must be such that all the material shown in the classroom is also shown in the video, be it lecture material or text written by the lecturer. This is important especially where the task of the lecture videos is, in addition to facilitating revision, make absences possible there.
One of the topics that merits consideration is whether to prioritize the image of the lecturer in the video or optimal visibility of lecture material. Prioritization of the lecturer's image poses some additional challenges to the production process, e.g., in deciding how to arrange the lighting.

One of the aims of video use is, typically, add flexibility to study. For this to occur, the video production must cover all the teaching provided as comprehensively as possible. The benefits of flexibility to the provider of education are questionable if they are related just to individual courses. The recording system used, and above all the practices constructed around it, must be able to manage with also larger volumes of video production while satisfying all other requirements set for it. Flexibility is also increased by that the videos are made viewable on as many technological platforms as possible. When planning video production, it is important to pay attention to the format the videos are produced in and whether to enable their viewing for example with mobile equipment. Changing the video format afterwards can be a fairly laborious and expensive process.

Typically, education providers do not have large financial resources at their disposal for the realization of video production. For this reason, one of the primary concerns in video production is cost-efficiency. Naturally, some costs are incurred by the infrastructure required. The costs due to equipment are nevertheless one-time costs only. Most of the costs are generated in the long term by the human resources required in the production. Minimization of man-days is largely based on automation and distance control of equipment.

To enable study with videos at all, they must be of good quality. However, as far as the quality of videos is concerned, some compromises might need to be made in order to satisfy other demands. If the quality is improved, in practice that means that more bandwidth is required for data communications. This, to some extent, limits the reachability of videos. Even then, it is nevertheless necessary to ensure that the quality remains sufficient for its purpose of use, that is, for study.

Once videos form an essential component for studies, video production must function properly. Reliability of production must always be kept in mind during development work. Reliability can be increased, for example by paying attention to backups. Recording of lectures must function well, regardless of problems in the data network for example.

\section{B. Technological Solutions}

In the development of a technological environment, choices that define the technologies must be made. These kinds of choices must be made, for example concerning the quality of image and sound and that of the video image itself. There will be questions such as whether it would be desirable to have an HD quality video image or whether, in addition to the lecture material shown in the video, we would also need the image of the lecturer or a general image of the class room. The technologies to be chosen are also determined by the requirements set for automation, transparency and file types to be produced.

To ensure comprehensiveness and cost-efficiency of video production, it is important to take video production into account already at the planning stage for the spaces required. 
From the lecturer's perspective, transparency in video production means that the lecturer can show with the projector any image source connected to the system at the class room and that the image from the source will be recorded. Also, it should be possible to automatically and without separate individual microphones record speech. Thus the lecturer needs to take only minimum care about the recording of sound and image.

When assembling the production equipment, it must be considered what the image sources that the lecturer should use are. Usually, at least a computer and a document camera are used as image sources. If a versatile system is wanted, an image source can also be realized through a connection to external video and image sources. If so desired, the system can also be connected to an interactive drawing board or terminal, which will function both as an image presentation equipment and as an image source.

It is also important to take into account transparency when recording speech. To prevent burdening the lecturer with the recording of sound, it is practical to use phantom-current powered microphones that are always kept on. In far-automated production, a situation where for example the battery of a microphone transmitter goes flat unexpectedly or it is forgotten to turn the microphone on cannot be tolerated.

Operation reliability for the production can be increased with a separate backup equipment. The backup equipment should be such that a recording of the video can be realized even when no direct transmission is possible due to network problems. Laptops of various kinds are practical for that role because their batteries can also help overcome short power outages. Devices inputting images and sound need, naturally, electricity to function and thus recording with them won't succeed during outages. The only purpose of the backup systems equipped with batteries is to ensure that once the power is restored the production will resume by itself.

Software used in the production includes a live media encoder, editor and media server and possibly also programs connected with timing and automatic data transmission. A possible inclusion among these programs is a media player used for viewing lecture videos. The task of the media server is to receive media flow material from the encoder and forward that material to the clients, in this case to media players. The choice of the software is influenced, above all, by the file formats to be produced.

\section{Production Process}

The administrative capacities of a media production system can be made more efficient by enabling distance control for computers and media flow servers that produce media flow. This is done with the help of a remote desktop, with which it is possible to affect the control through Internet and a VPN connection. The setup enables a fast reaction in problem situations when needed. However, in physical problem situations a visit to the location is always required.

A production process can be divided into preliminary arrangements, recording stage, distribution and post-production. These stages and the actions related to them are summarized in Fig. 1.

\section{1) Preliminary arrangements}

Timing of the start and end for a recording should be done well before the recording itself takes place. Combined with the timing operation, it is also possible to define, for example, the bandwidth, frame rate and size and other settings related to the compression of image and sound.

Transfer of the recorded raw version to the media flow server is best achieved with automated program code. During the preliminary arrangements, the transfer can be timed to be carried out once the lecture has ended. Depending on the distribution methods, linkages required by the distribution can be made, for example, to the LMS's or WWW pages, at least as far as real-time video is concerned but probably also for recordings. In this way, the recorded videos will be available to students immediately after the automatic file transfer.

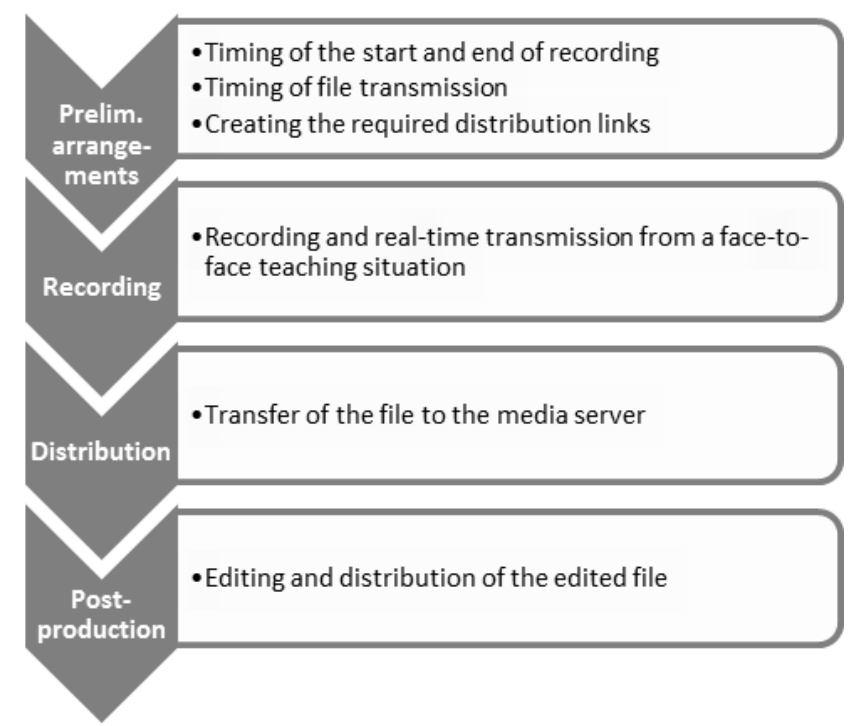

Fig. 1. The stages in the production process of lecture videos.

\section{2) Recording stage}

In an automated system, the image shown with a projector by the lecturer is automatically directed from the image source to the recording equipment. If the system is not automated, the lecturer must select the source to be recorded. This clearly reduces the transparency and reliability of production.

The media encoder saves a local version of the teaching session and, using push technology, transmits the compressed material further to the media server. Also the media server can be configured to store a backup copy of the material. That copy can then be retrieved for use in case the indexing of the recording saved by the media encoder fails or is damaged.

\section{3) Distribution}

Real-time media flow is transmitted from the media server to the clients by using unicast or multicast technologies. The unicast solution is appropriate if the number of simultaneous viewers is moderate enough not to put excessive strain on the network's active devices. In other cases, it might be better to think about using a multicast technology or Content Delivery Network solutions. Distribution of on-demand videos to students should be carried out as soon as possible once the recording has been made. The delay in the distribution can be minimized by realizing the transmission of the lecture recording from the encoder to the media server automatically 
with the help of program code and by timing it to take place as soon as possible after the lecture to be transmitted as a media flow has ended.

\section{4) Post-production}

Depending on the needs, the recordings can be edited. In a cost-efficient solution, only very small amount of editing is done to the recordings by, for example, cleaning up extra material from the beginning and end of the raw version of the recording. The cleaned-up version replaces the unedited version of the recording automatically transferred to the media server. For editing, it is best to use a program that does not recompress the material, which guarantees that the quality of the material will remain unchanged.

\section{Video Production OF THE MASTER StUdies IN MATHEMATICAL INFORMATION TECHNOLOGY AT THE KOKKOLA UNIVERSITY CONSORTIUM CHYDENIUS.}

Lecture videos have been produced since 2002 in connection with the master's studies in mathematical information technology, and the number of video transmissions has been increasing constantly. Currently, the annual volume of teaching sessions transmitted as real-time video amounts to hundreds, and the viewing time per student reaches dozens of hours. Lecture videos form, thus, an essential element of the education arrangements for the master's studies in information technology.

Production of videos for these studies follows the model presented above. The videos are produced from face-to-face teaching and offered both as real-time and on-demand videos to students. The primary considerations for the production are, above all, availability, transparency, good visibility of lecture material and cost-efficiency. These considerations also include that the video production must cover all the teaching of the degree program.

Cost-efficiency is supported by the fact that face-to-face teaching is paced over and assembled over two days per week This allows many of the operations, such as timing of the recording that is applied to several lectures simultaneously. To reach the goals, attention is paid to software that supports the practices as well as to technical and quality-related solutions in video production equipment.

From a technological perspective, the basic idea for the video production of the master's studies in information technology is that the signals from image sources are distributed to the video production systems, prior to a signal reaching the projector. In this way, all the material shown by the projector in the class room is recorded on the video. Therefore, it is enough that the lecturer knows how to bring the desired image to the view in the projector at that class room. In this solution, the core of the video production equipment is the media presentation switcher where the image and program sound inputs are directed to. The media switcher's image signals are guided through the required distributors and converters to the recording and backup systems and to the projector. The program audio sources from the switcher and speech from the microphones are directed through an audio mixer to the recording and backup systems. The system similar to the one described above is presented in Fig. 2.

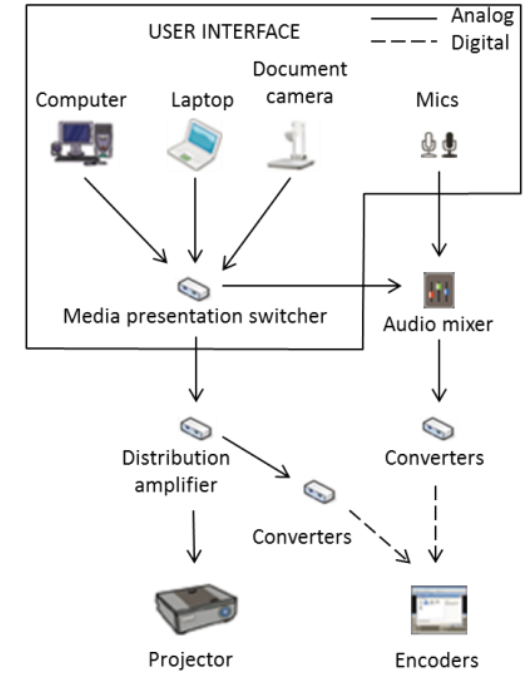

Fig. 2. The equipment making up the storage system for the master's studies in information technology.

The production takes place in Windows Media environment. The media server implementation is based on the Streaming Media Server -role of the Microsoft Windows Server 2003 Enterprise Edition. Windows Media Encoder is used as the encoder. The timing of the encoder's file templates is realized with the graphical Scheduler timing program available in the Windows Media Software Development Kit, which utilizes the Windows operating system's own Task Scheduler function. Post-production is done with the Windows Media File Editor program that accompanies the Windows Media Software Development Kit.

To view the videos, students need an active Internet connection and user rights. Videos can't be downloaded by students. With the help of user rights, the video distribution can be limited only to the students who have enrolled in the course. The videos are removed from distribution once the course has ended. User rights and disposability of videos have helped us to avoid problems related to copyright and intellectual property. In addition, user rights also make it possible, in problem situations, to search for user-specific solutions and to conduct research on utilization of videos. Maintenance of user rights for videos does not create extra work because it is carried out with the help of user rights of LMS's courses.

\section{Evaluation of the Production Model And ASSESSMENT OF THE EFFECTIVENESS OF ITS TECHNOLOGIES}

The research data was collected in connection with the Master Studies in Mathematical Information Technology at the Kokkola University Consortium Chydenius. The material was obtained from the data of the university consortium's space reservation system, from the 2010 structured interviews of master's studies' students, from the accounting of the education provider about failed events as well as from the media flow server's log data and its 2008-2011 attendance records.

\section{A. Suitability for the Purpose}

The students of the degree program can freely choose their mode of participation in each lecture between face-to-face 
study, real-time video and on-demand video. The suitability of lecture videos for their intended purpose can be examined by comparing the numbers of those participating with the help of lecture videos to those participating with the help of face-to-face teaching. One would think that possible unsuitability of lecture videos would be reflected in their popularity ratings.

Fig. 3 shows the shares of the students' participation modes in all lecture participations during 2008-2011. The data includes 30 courses for which attendance records have been collected. A total of 80 master's degree students participated in these courses, adding up to a total of 2988 lecture participations. The important role of videos in study participation can clearly be seen from the figure. When the students were completely free to choose their participation mode between face-to-face learning and videos, they gravitated towards participation with the help of videos.

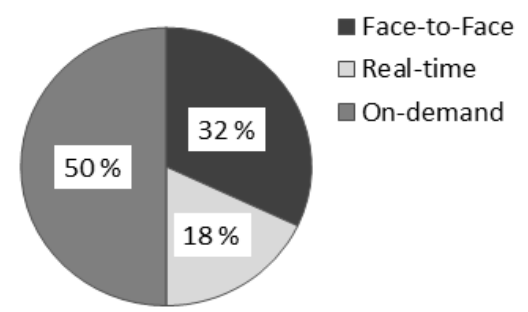

Fig. 3. Relative shares of participation modes of all lecture participations 2008-2011 (n=2988).

Student's opinions also reflected the suitability of videos. According to a structured questionnaire survey of students carried out in 2010 , only $4 \%$ of the students felt that lecture videos (real-time or on-demand) made learning more difficult The questionnaire was responded to practically by all active students at that time $(n=67)$.

Videos' effectiveness in study can also be evaluated from the perspective of learning outcomes. The impact on learning outcomes in connection with master's studies in information technology was examined separately in an earlier research [10]. When the learning outcomes were examined with the help of completion performance and grades, the research found that participation with the help of videos had a positive effect on students' learning outcomes. Videos enabled an increase in the degree of participation, which in turn improved the course completion figures and grades. The results were in general agreement with the results of research carried out elsewhere, e.g. [1], [2], [11], [12].

\section{B. Transparency}

One of the starting points in video production has been its transparency for the lecturer and students. In practice, recording itself does not distract the lecturer. The lecturer doesn't need to worry about starting the recording or stopping it, for example. The only occasion when the lecturer needs to change his/her habits of acting is when the whiteboard is replaced with a document camera. However, the lecturer must be able to control the technical infrastructure of the class room, either through one's own know-how or with the help of the written instructions and personal operation practice devised on behalf of the education provider, One should remember, however, that some of this equipment would be used in the class room even without any recording taking place. For the students participating in face-to-face study, the production process is transparent. Video production does not disturb face-to-face study, and, according to earlier research, for example the document camera is regarded by the students as even a better alternative than the whiteboard [13].

\section{Cost Efficiency}

The costs of the video production for the master's study in information technology consist of initial costs, development costs and operation costs. The initial costs such as acquisition of equipment are one-time costs, and their magnitude depends on, e.g., the quality of materials wanted.

When the system is self-implemented utilizing and combining various commercial equipment and software solutions, development work can be carried out step by step from the standpoint of economy. Big one-time investments won't be necessary. In this kind of system, it is easy to integrate add itional parts as needed with the advancement of the development work. The extra parts required can be self-constructed, and commercial solutions may be considered if they have interfaces to connect them as parts to the existing environment.

However, in the long term, the ability to control operation costs is important as far as cost efficiency is concerned. Cost efficiency in the case of operation costs is based on task automation, remote equipment control and planning of the teaching schedule by the education provider.

Timing of education can have a great impact on operation costs. As the teaching in the master's studies in information technology is spread over two subsequent days, the operations related to the information flow can be executed concurrently and the work won't need to be distributed over several days. In practice, all the video production arrangements for teaching during a weekend take about two man-hours. This time is divided fairly equally between preliminary arrangements and light editing of the material produced. Video production carried out for the master's studies in information technology takes slightly below 100 man-hours on an annual basis.

The cost-efficiency of operation costs is emphasized in the initial arrangements for video production and in post-production because these stages require most man-hours. One should, nevertheless, bear cost efficiency in mind also when dealing with matters related to distribution, quality control and backup.

\section{Operational Reliability}

Hundreds of hours of lecture videos are yearly produced in connection with the master's studies in information technology. Compared with the total number of recorded lectures, complete failures (e.g., a lecture couldn't be viewed in real time and no recording could be made) are remarkably rare. Even the number of partial failures (a real-time lecture transmission could not be realized or it was necessary to resort to a poorer quality backup recording for later viewing) was only slightly higher,

Table I shows the number and duration of the lecture videos in the master's studies in mathematical information technology and the number of complete and partial failures during 2008-2011. The table presents matters related to the recordings of face-to-face study sessions but not the 
problems related to the viewing of recordings. The table shows that the annual number of all failures is about $4 \%$ and that the number of complete failures is even smaller (approx. $1 \%)$. For example, in 2011 there was only one complete failure.

TABLE I: NuMBER OF LECTURE RECORDINGS, THEIR DURATION AND THE DOCUMENTED FAILURES

\begin{tabular}{|l|c|c|c|c|}
\hline & $\mathbf{2 0 0 8}$ & $\mathbf{2 0 0 9}$ & $\mathbf{2 0 1 0}$ & $\mathbf{2 0 1 1}$ \\
\hline No. of lecture recordings & 145 & 215 & 185 & 164 \\
\hline Duration/hr & 453 & 707 & 635 & 518 \\
\hline No. of complete failures & 2 & 2 & 2 & 1 \\
\hline No. of partial failures & 7 & 5 & 6 & 4 \\
\hline
\end{tabular}

A complete failure in the recording of a lecture video can have various reasons behind it. The most probable of these reasons are lack of timing in the encoder or its incorrect timing, failed activation of a timed task and power failure. In the production model of the master's studies in information technology, complete failures have always been due to these factors. For partial failures, there are numerous reasons. In practice, the reasons are the same as in complete failures, but they take place in a more reduced scale. Examples include the cases where an encoder transmitting real-time media flow or an encoder recording it cannot capture the image input. This may be due to the breaking of the hardware or the computer has turned itself off as a result of a power outage. Nevertheless, the backup system has saved the lecture.

It can be stated, therefore, that the problems with lecture videos are related to preliminary arrangements and recording as well as to external factors such as power and equipment failures.

\section{CONCLUSIONS}

Production of lecture videos is a topical issue for many of those who provide modern education. Often there is reluctance to give up face-to-face teaching, and for the reasons of cost efficiency there is preference towards video production connected to face-to-face teaching situations. Production circumstances of this kind pose many challenges to video production.

The technological environment required in video production is fairly simple. However, integration of a production system to existing systems, automation taken to as far as possible, transparency of production and wide reachability of videos are quite demanding to accomplish. In addition videos are often solicited simultaneously from several locations and production processes should be suitable for different courses and lecturers. These kind of demands placed on production diversity create an additional challenge. In many cases, challenges are easier to meet with the help of a self-constructed production system that utilizes various commercial equipment and software solutions than with the help of a comprehensive commercial recording system.

The more we want to take into account the demands presented above, the more technological environment we must build by ourselves. From the perspective of cost efficiency, the practices developed around technological solutions are more important than the solutions themselves.

The research results indicate that lecture videos are suitable for the purpose they are intended. In practice, this means that with their help availability of teaching can be improved and, through this, students' learning can be supported. Apart from the increased flexibility, videos offer also other benefits such as opportunities for revision and support for different learning styles.

By individual construction - utilizing, combining and supplementing equipment and software on the markets to obtain a technological environment that is just right for the practices and needs of the education provider - it is possible to create a production model that enables a very cost-efficient way to produce lecture videos. Based on the results, it is possible to assert that the operational reliability in systems built in this way can be brought to a quite reasonable level. One should keep in mind, however, that creating this kind of recording system requires that technological know-how can be found in the organization.

\section{REFERENCES}

[1] I. N. Toppin, "Video lecture capture (VLC) system: a comparison of student versus faculty perceptions," Education and Information Technologies, vo. 16, no. 4, pp. 384-395, 2011.

[2] G. Preston, R. Phillips, M. Gosper, M. McNeill, K. Woo, and D. Green, "Web-based lecture technologies: highlighting the changing nature of teaching and learning," Australasian Journal of Educational Technology, vol. 26, no. 6, pp. 717-728, 2010.

[3] S. Engstrand and S. Hall, "The use of streamed lecture recordings: patterns of use, student experience and effects on learning outcomes," Practitioner Research in Higher Education, vol. 5, no. 1, pp. 9-15, 2011.

[4] H. Brecth and S. M. Ogilby, "Enabling a comprehensive teaching strategy: video lecture," Journal of Information Technology Education, vol. 7, pp. 71-86, 2008.

[5] M. Gosper, M. Mcneill, R. Phillips, G. Preston, K. Woo, and D. Green, "Web-based lecture technologies and learning and teaching: a study of change in four australian universities," Research in Learning Technology, vol. 18, no. 3, pp. 251-263, 2010.

[6] K. T. Tan, E. Wong, and T. Kwong, "Piloting lecture capture: an experience sharing from a Hong Kong university," Enhancing Learning Through Technology, vol. 117, pp. 268-279, 2010.

[7] A. Le, S. Joordens, S. Chrysostomou, and R. Grinnell, "Online lecture accessibility and its influence on performance and skills-based courses," Computers and Education, vol. 55, no. 1, pp. 313-319, 2010.

[8] F. Bodendorf, M. Schertler, and E. Cohen, "Producing Reusable Web-Based Multimedia Presentations," Interdisciplinary Journal of Knowledge and Learning Objects, Vol. 1, pp.127-142, 2005.

[9] R. Kannan and F. Andres, "Towards automated lecture capture, navigation and delivery system for web lecture on demand," International Journal of Innovation in Education, vol. 1, no. 2, pp. 204-212, 2010.

[10] I. Hakala and M. Myllymaki, "The use of lecture videos: attendance and student performance," in Proc. 14th International Conf. on Computers and Advanced Technology in Education, Cambridge, United Kingdom, 2011.

[11] M. Wieling and W. Hofman, "The impact of online video lecture recordings and automated feedback on student performance," Computers \& Education, vol. 54, no. 4, pp. 992-998, 2010.

[12] T. Traphagan, J. V. Kucsera, and K. Kishi, "Impact of class lecture webcasting on attendance and learning," Educational Technology Research and Development, vol. 58, no. 1, pp. 19-37, 2009.

[13] I. Hakala and M. Myllymäki, "Video lectures alongside with contact teaching," in Proc. $18^{\text {th }}$ Annu. EAEEIE Conf. Innovation in Education for Electrical and Information Engineering, Praque, Czech Rebublic, 2007.

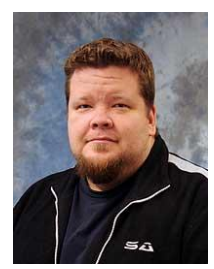

Mikko Myllymäki received his master of science in information technology from the University of Jyväskylä in 2008. He is currently working as a researcher at the University of Jyväskylä and is finalizing his Ph.D. thesis. His research interests include learning technologies and especially impacts of the use of these technologies. 


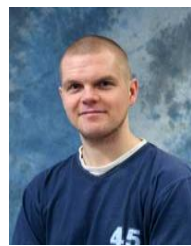

Jari Penttilä is a student at the University of Jyväskylä $\mathrm{He}$ is currently working as a planner at the Kokkola University Consortium Chydenius and he is currently finalizing his Master's thesis, which deals with thematic areas of this paper.

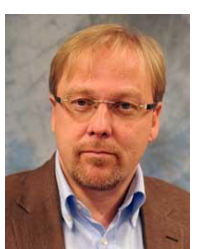

Ismo Hakala received his $\mathrm{Ph} . \mathrm{D}$. in mathematics from the University of Oulu in 1997. He is currently working as a professor of computer science at the University of Jyväskylä, Finland. He works also as a Head of Information Technology Unit at the Kokkola University Consortium Chydenius. 